Fecha de recepción: diciembre 2018 Fecha de aceptación: marzo 2019 Versión final: abril 2019

\section{El diseño representado a través de la artesanía. Emprendedores de la etnia Mapuche. Región de la Araucanía, Chile}

Eugenia Alvarez Saavedra *

Resumen: En el siguiente artículo se presenta una síntesis de la investigación realizada en el marco del Doctorado en Diseño de la Universidad de Palermo, Buenos Aires, Argentina. El estudio es referido al desarrollo de la artesanía en emprendedores de etnia Mapuche en la región de La Araucanía, Chile. El abordaje se orienta al estudio de la relación que tiene el trabajo en diseño con motivos gráficos en materiales y formatos que permite la elaboración de productos artesanales. Materiales como el cuero, la plata, la lana, la cerámica greda y la madera son algunos de los objetos de estudio que se presentan en las propuestas de los emprendedores que diseñan motivos gráficos en ellas.

Palabras clave: Emprendimiento - Diseño - Mapuche - Etnia - Artesanía

[Resúmenes en inglés y portugués en las páginas 217 - 218]

${ }^{*}$ Académico, Docente. Universidad Católica de Temuco, Chile.

\title{
Introducción
}

En la región de La Araucanía, Chile, se sitúa la etnia más importante del país, denominada Mapuche, los cuales son considerados descendientes directos de las culturas arqueológicas prehispánicas Pitrén (100 - 1100 años d.C.) y El Vergel (1100 - 1450 años d.C.), que se desarrollaron en la región, entre el río Bío Bío y el seno de Reloncaví. La llegada hispana en el siglo XVI hizo que poblaciones originarias distintas se agruparan y estrecharan sus lazos sociales y culturales, formándose la identidad mapuche conocida históricamente. Los inicios al sometimiento del pueblo Mapuche culminaron ante el Ejército de la República de Chile con la así llamada Pacificación de la Araucanía, en 1882.

Lo mencionado anteriormente son aspectos esenciales propios de la cultura Mapuche, basados en la pasión, energía y amor por su tierra, afectada directamente en esos tiempos por la llegada de colonizadores violentos. La historia, de alguna forma, va diseñando estos aspectos propios de la etnia Mapuche, resaltando la defensa, la libertad de acción, la pasión, la exaltación y la bravura de sus acciones en respuesta a la provocación y ocupación de sus territorios. 
La investigación en diseño se fundamenta en el emprendimiento de la etnia Mapuche en la región de la Araucanía, Chile. Se hace luz directa en estos trabajadores que se apegan a un modelo de negocios con el fin de comercializar sus ideas e ingresar en un mercado regional delimitado por la reunión de emprendedores Mapuche de distintas zonas de la región de La Araucanía. Puntualmente, el análisis de las representaciones con motivos gráficos presentes en los diferentes formatos que los emprendedores diseñan, son el foco de la investigación, la relevancia de cada uno de esos relatos gráficos, su comunicación, forma y color.

Se presentan diez casos de estudio, basados en la aplicación de entrevistas semi estructuradas y abiertas a emprendedores que trabajan la madera, lana en telar, cuero, orfebrería en plata y greda. Cada uno de ellos presentan sus creaciones, con la singularidad que deben tener en el diseño la simbología Mapuche, a través del trabajo artesanal desarrollado en la zona de estudio.

\section{Artesanía Mapuche}

La artesanía, como producto folklórico, ha conformado rasgos distintivos de la identidad, como individuos y como colectivo. Ese proceso ha estado determinado por el medio ambiente y la realidad cultural, social y económica. Las creencias, artes, valores, prácticas y tradiciones que se transmiten de generación en generación, sugieren una memoria que vive el presente poniendo en valor las experiencias ancestrales en la cotidianidad de su quehacer (Navarro Hoyos, 2017). Como indica el autor, es importante el rescate de los valores y creencias del artesano, ya que tiene la capacidad de poder sintetizar los rasgos identitarios de su cultura y plasmarlos en una pieza de diseño. Haciendo énfasis también en el hecho de comercializar este producto o servicio que ha realizado, foco de interés en el análisis de este trabajo.

La artesanía se define desde tres dimensiones: primero el artesano, como creador y constructor de cultura. En segundo lugar la actividad artesanal, como proceso en el que se aplican técnicas y prácticas artesanales tradicionales y contemporáneas y como proceso productivo que provee medios de vida al artesano. Por último el producto artesanía, como expresión de identidad y de la cultura autóctona nacional, regional y local. (Navarro Hoyos, 2017). A nivel internacional, la UNESCO define la artesanía de la siguiente manera:

Los productos artesanales son los producidos por artesanos, ya sea totalmente a mano, o con la ayuda de herramientas manuales o incluso de medios mecánicos, siempre que la contribución manual directa del artesano siga siendo el componente más importante del producto acabado. Se producen sin limitación por lo que refiere a la cantidad y utilizando materias primas procedentes de recursos sostenibles. La naturaleza especial de los productos artesanales se basa en sus características distintivas, que pueden ser utilitarias, estéticas, artísticas, creativas, vinculadas a la cultura, decorativas, funcionales, tradicionales, simbólicas y significativas religiosa y socialmente. (UNESCO 1997). 
Esta definición resulta adecuada para la propuesta de estudio, ya que considera aspectos relevantes que han sido verbalizados por los mismos emprendedores - artesanos Mapuche, de alguna forma ellos buscan plasmar o reflejar el sentimiento de cultura, identidad y religiosidad en sus creaciones, para finalmente poder ofrecerlas en un mercado determinado. Así se desarrolla el proceso de comercialización propuesto por los emprendedores locales, generando un mercado regional y sobre todo turístico en las zonas de estudio de la investigación; Pucón, Temuco y Villarrica específicamente. Al hablar del producto artesanía, está implícito su comercialización y su apertura a mercados, y por lo tanto está influenciado por un consumidor, es quien al final hace que el objeto cotidiano devenga en artesanía (Navarro Hoyos, 2017). Como indica el autor, la comercialización actual en la región, tiene una tendencia a adaptarse a las demandas específicas, a modo de respuesta a lo que el grupo objetivo desea. Los emprendedores de etnia Mapuche son orgullosos de sus creaciones, y se presentan como empresarios del rubro, ya sea desarrollando trabajos en madera, lana, greda, orfebrería y cuero. Cada uno de ellos con el trabajo de especialidad que se menciona a continuación: madera, telar mapuche, textilería, cerámica, cuero y orfebrería .

\section{La Madera}

La madera como material ha sido trabajada a lo largo de la historia Mapuche como ornamento, ya sea tallada en base de dos dimensiones, así como en esculturas tridimensionales. Por ejemplo el diseño de utensilios de madera. La mayor parte de los objetos de uso diario en una ruka mapuche exceptuando las vasijas de cerámica eran confeccionados en madera. Estos objetos siguen siendo usados en muchas funciones en la actualidad, aún cuando existen alternativas de plástico y metal.

En las regiones de la Araucanía, de Los Ríos y Los Lagos, por otro lado, se desarrolla una importante actividad forestal y, por consiguiente, es el lugar donde existe la mayor producción artesanal asociada a esta materia. Es interesante apreciar la profusión de la artesanía en madera desarrollada en la Novena Región, especialmente en Malleco y Cautín donde se han elaborado una gran variedad de artefactos utilitarios de decoración y muebles tallados, utilizando principalmente la madera de raulí y mañío. En la comuna de Villarrica se concentra la mayor oferta de estos utensilios.

También los Mapuche trabajan el tallado en madera, que es una de las manifestaciones artesanales con mucha tradición en la zona, con esta técnica elaboraron utensilios los antiguos habitantes de estas tierras, utilizando para ello las maderas de los ricos bosques del sector. En la actualidad se sigue trabajando de la misma manera, con el hacha y azuela tallando la madera pero creando nuevos productos, algunos de tipo utilitarios como platos, cucharas y cucharones, fuentes, bandejas etc y otros en que el uso se complementa con la decoración, por ejemplo en el diseño de flora y fauna del sector.

Este tipo de trabajo transita entre la funcionalidad doméstica y el poseer un significado ritual, pues las máscaras que representan al Kollom cumplen un rol específico en la ceremonia del Nguillatun. Por ejemplo el rehue y el chemamull son troncos tallados que rematan en amplias cabezas. La máscara ritual, el kollón, se complementa con pelo de 
crin de caballo. Es importante mencionar que los emprendedores trabajan este material tanto como en el diseño de elementos de ornamentación, útiles de distinta índole, incluso armas, y hacia la funcionalidad en el trabajo de mueblería. En todos los casos anteriores se encuentran emprendedores de la región que plasman con distintas técnicas extractos de la simbología Mapuche.

\section{El Telar Mapuche}

En el caso del trabajo en lana, se ha investigado por un lado a las emprendedoras de telar, y por el otro a quienes realizan diseño de indumentaria elaborado con lana. La técnica específica del telar, se desarrolla con lanas puras, teñidas y preparadas para la confección final y es desarrollada por las emprendedoras Mapuche en la zona de la Araucanía. La fundación Chol-Chol es la que reúne la mayor cantidad de creadoras de telar, exhibiendo sus creaciones en el sitio web de la corporación y así ayuda en la difusión.

El tejido en telar mapuche es una tarea exclusivamente femenina que si bien está destinada al uso cotidiano, esconde en sí misma una simbología solo conocida por las grandes tejedoras. Antiguamente la gente se tejía su ropa para vestirse, para salir o para asistir al lepún. Los tejidos se hacían en telares, los cuales se construían con madera de distintas medidas de acuerdo al tamaño de la prenda. El telar está formado por una kilwa, que es un palo atravesado donde va la lana; el parampawe, que es una tablilla que baja la cruzada de la lana; el ñirewe, para apretar el tejido que se hace con madera de luma porque es más pesada y dura y por el tonón, un palo fijo que se encarga de que la lana se vuelva a cruzar y va acompañado de unos coligües que lo levantan.

La esquila de las ovejas se debe realizar antes del veinte de diciembre. La lana obtenida se lava bien, se deja unos días al sol y después de lavarla nuevamente, ya está lista para tejer. (Inés Panguilef, 1999). En el caso mencionado de la indumentaria, las Mapuche trabajan la lana pero la denominan textilería.

\section{La Textilería}

En la textilería Mapuche, la diferencia entre los diversos tipos de tejido, no está en su destino o utilidad, sino en el gran simbolismo de sus contenidos. La textilería mapuche está desarrollada exclusivamente por las mujeres. La enseñanza se transmite de madre a hija, como parte final de un proceso de aprendizaje general. Antes de la ejecución de una pieza textil, ésta debe ser pensada en un sentido técnico y simbólico. En su concepción, en primer lugar, priman las técnicas de elaboración, en segundo lugar los contenidos culturales y finalmente lo estético (Soto, 1996). La elaboración de un textil tradicional se realiza a través de un largo proceso que se puede subdividir en las siguientes etapas:

Lavado de la lana obtenida en la esquila. Ésta se lava prolijamente con agua caliente, extrayendo todos los restos orgánicos que se van adhiriendo a ella a lo largo de la vida de la oveja. Luego se enjuaga con agua fría y se trabaja el hilado. 
Secado de la lana. Una vez que la lana está limpia, se deja estilar y se deposita sobre una superficie plana para que se seque al sol o cerca del calor de la cocina o fogón.

Escarmenado de la lana. Este trabajo consiste en estirar los fragmentos de lana esquilada, separando a mano cuidadosamente las fibras sin que se corten hasta que adquieran una textura suave y un peso muy liviano.

Hilado de la lana. Con su huso girando en torno a la tortera van produciendo hilos de distinto grosor, dependiendo de la prenda que piensan fabricar.

Madeja. Se recoge la lana hilada en vueltas iguales con un aspa para crear una madeja, la que se lava nuevamente con jabón para eliminar todo tipo de residuos que aún estén en ella. Después de este lavado, se utiliza en su color natural o se tiñe con el color deseado.

Teñido de la lana. Se selecciona el producto vegetal del que se desea obtener el color para teñir la lana. Se hierve en agua hasta lograr que desprenda el color. Se agrega la lana mojada enmadejada. Finalmente, después de un período de cocción, se agrega una sustancia que fija el color (sal, vinagre, piedra lumbre o sulfato de cobre).

Tejido de la lana. Cuando la lana teñida está seca y el telar ha sido preparado según las medidas de la prenda que se elaborará, la artesana inicia el tejido urdiendo la lana en el witral o telar y, gracias a su particular experiencia, entrelaza las hebras y da origen a un producto único. (Aedo, 1992).

El huitral o telar araucano es un aparato sencillo que consta esencialmente de un marco rectangular de madera formado por cuatro palos más o menos derechos que se cruzan en ángulos rectos. Estos palos están sólidamente amarrados en los cruces de los cuatro ángulos con cuerdas de junco trenzado o con tiras de tejido. Los dos palos más largos que alcanzan a dos metros y medio a tres están destinados a soportar todo el peso del telar, apoyados en sus extremos más gruesos en el suelo y por el otro contra el techo de la ruca. Los dos palos atravesados encima del huicha huichahue, uno en la parte superior y otro en la parte inferior, labrados con más cuidado, de forma cilíndrica, estás destinados a recibir los hilos de la urdimbre.

\section{El trabajo en greda}

El trabajo elaborado en greda por los Mapuche consiste en moldear el barro para luego cocerlo. Este material se trabajó en los pueblos originarios para resolver necesidades básicas de la etnia y sobre todo en ceremonias. En el pasado pre-hispánico, los pueblos de América tenían un nivel de desarrollo social y cultural que se puede apreciar en los testimonios materiales encontrados. El uso de la arcilla en piezas ceremoniales y utensilios domésticos, alcanzó una perfección técnica y estética notable.

El patrimonio dejado por los pueblos indígenas se fue transformando. Ello, unido a la presencia de España, con toda su carga cultural, ha generado una expresión propia de estos pueblos que muestran en su arte popular y su artesanía tradicional, el reflejo de lo que fueron y lo que son. (Roselló, 2009). Las técnicas de greda se desarrollan actualmente en cinco zonas bien caracterizadas: Pomaire, Talagante (área de Santiago); Quinchamalí (área de Chillán); Florida (área de Concepción), y localidades en muy pequeñas de la cultura mapuche en zonas de la Araucanía, como Pucón y Villarrica. 
En la actualidad, es en la provincia de Cautín donde están más a la vista los grupos mapuches que elaboran cerámica vinculados al comercio con Temuco. La mayoría de la cultura material mapuche se produce actualmente con finalidad turística, aunque se siguen fabricando algunos objetos para cubrir algunas necesidades propias. Quedan todavía casas mapuches (rukas) familiares donde, junto a la agricultura elemental, se elaboran cerámicas, tejidos y cestería. La gente de la ciudad compra los objetos más elaborados, generalmente zoomorfos, pero la producción doméstica se fundamenta en la fabricación de vasijas no muy grandes como cántaros, callanas o jarros para el muday, bebida fermentada hecha del piñón del calafate o maíz.

Cabe aclarar que específicamente la greda se clasifica dentro del concepto cerámica, dado que la técnica es similar pero con sustitución de materias primas. Los Mapuche trabajaron la greda en sus inicios como pueblo originario, pero actualmente el desarrollo en propuestas de cerámica es importante, tanto así como la materia prima inicial, la greda. Es por esto que la técnica está presente en distintas localidades a lo largo de Chile.

\section{El trabajo en cuero}

El trabajo en cuero fue desarrollado por los pueblos originarios desde sus inicios de forma artesanal para el cobijo y seguridad en la vivienda. Diseñaron su vivienda y artículos en cuero, con la técnica de la talabartería. La guarnicionería o talabartería es el arte de trabajar diversos artículos de cuero o guarniciones para caballerías. Se considera guarnición a todos los elementos de la espada que sirven para sostenerla o para proteger a la mano o manos que la empuñan, así como a la fabricación o arreglo de sillas de montar de caballería, albardas y aparejos. Se suelen usar otros materiales además del cuero, como la lona, lanas gordas denominadas estambre con las que se elaboran las guarniciones o dibujos sobre las monturas o aparejos e hilos de colores (Cogollo Arrieta y Valencia García, 2013). La palabra talabartería designa el trabajar la piel, transformarla y darle un sentido útil y bello. El trabajo en cuero en la actualidad está relacionado directamente con la ornamentación, y funcionalidad, por ejemplo los diseños de indumentaria, muebles y accesorios. Los emprendedores que trabajan este material diseñan sus artículos tanto para mujeres como para hombres, y se adaptan a la demanda local.

\section{La orfebrería o Platería Mapuche}

La presencia de la metalurgia en el pueblo mapuche data de la época prehispánica. Sin embargo, la orfebrería en plata adquirió gran relevancia al interior de la comunidad mapuche, a fines del siglo XVIII, comenzando a ser desarrollada en forma intensiva por hábiles artesanos que lograron crear una gran diversidad de formas y estilos.

La gran cantidad de monedas de plata procedentes del activo comercio en la Frontera comenzaron a ser utilizadas como materia prima por los artesanos especializados de caciques y poderosos hombres de la sociedad mapuche. En vez de utilizarlas como valores de inter- 
cambio, los orfebres martillaban y fundían las monedas para luego confeccionar alhajas para las mujeres y diferentes artículos usados en los arreos y aperos de los jefes, también llamados cacique o longkos. De esta forma, la platería obtuvo un importante valor como símbolo de status para longkos y ulmenes, y para sus mujeres que llevaban puestas estas joyas con sus atavíos rituales y festivos. Durante el siglo XIX, período de auge de la platería mapuche, se multiplicaron las formas de las piezas y sus diseños y decoraciones. No obstante, este fuerte desarrollo en la orfebrería decayó con el proceso de ocupación de la Araucanía.

En el trabajo en metal, la cultura mapuche aprovechó las monedas para crear joyas además de platos, vajillas y mates. Este trabajo nació a fines del siglo XVIII y en la actualidad sobreviven las formas tradicionales como el trarilonco, los sükill y las trapelacucha, los chaway, el tupu que trabajan los retrafe (platero mapuche) (Chávez, 2013). La platería fue una de las expresiones más observable del dinamismo y bonanza alcanzada por la economía mapuche en el periodo preocupacional, particularmente producto del comercio ganadero. La confección, evolución y acumulación de piezas de plata constituyó una expresión de la riqueza y poder alcanzado por caciques y ulmenes. Se asume, según la búsqueda bibliográfica sobre historia del pueblo Mapuche, que la pérdida de estas piezas de plata fue expresión de la precariedad económica por la que comenzó a transitar el pueblo mapuche en el proceso de colonización.

En los últimos años se han realizado miniaturizaciones de estas piezas con un carácter más comercial. Antes del trabajo en metal, la tradición de la cultura mapuche estuvo ligada al trabajo en piedra, cuando originariamente realizaban piezas denominadas hoy como "joyas líticas", asociadas a insignias de mando y llamadas "clavas". Además se hacían pipas, pitos y pifülcas con piedras finas en distintos colores (alabastro blanco, rojo, etc.) (Chávez, 2013).

Dentro de los materiales que trabajan los Mapuche, específicamente la plata, es uno de los más numerosos en el sector. En la actualidad, sobre todo en la Región de la Araucanía, sigue presente la tradición orfebre entre los plateros. La producción se dedica mayoritariamente a reproducir los sofisticados diseños ancestrales de formas antropoformas, fitomorfas, ornitoformas y geométricas, en reducidas proporciones. Lamentablemente las piezas relacionadas a lo ecuestre y a los objetos cotidianos, hoy ya no se producen.

Sobre lo relevado en la búsqueda bibliográfica y sobre todo en respuesta de entrevistas con emprendedores que pertenecen a fundaciones regionales, la historia menciona la pérdida de éstas prácticas en el periodo de ocupación de la Araucanía. Después de las incursiones militares iniciadas en la década del sesenta, que tuvo sus momentos más intensos entre 1868-1872 con la llamada "guerra de recursos" y en 1881 con el levantamiento general, la situación de los mapuches pasó a un estado de verdadera miseria.

Las investigaciones efectuadas por Walter Reccius, uno de los más reconocidos estudiosos de la platería mapuche, le permitieron afirmar que antes de la llegada de los españoles, los mapuches conocían el uso de los metales y manufacturaban adornos de cobre, al igual que de oro y plata (Chávez, 2013). Lo antedicho se contrapone con lo sostenido por Tomás Guevara (1925) y Arturo Fontecilla (1946) quienes afirman que el desarrollo de la platería entre los mapuches sería con posterioridad a la llegada de los españoles, quienes habrían introducido a los indígenas en este oficio. Es importante de alguna manera documentar y dar data del trabajo en orfebrería, ya que se maneja información errada sobre los inicios 
de la producción de elementos en este material. Con el pasar de los años, las actividades de desarrollo en orfebrería se han ido retomando, y sobre todo el trabajo de la platería, por lo cual las nuevas generaciones han ido adaptándose al mercado regional, diseñando nuevas propuestas para la creación de joyería.

\section{El uso del color en las representaciones con motivos gráficos}

Los colores son fundamentalmente luz para la cultura mapuche. Esta designa con el mismo lexema al color blanco y a la luz: lig. Además, el término kuri designa al color negro y a la ausencia de luz, la oscuridad (Alvarado, 2004).

Color y luz logran una síntesis de significado por medio de la homonimia, que obliga a considerarlos a ambos en su ambivalencia significante para su comprensión. El matiz, la luz que posee el objeto (no hay refracción en este caso para la física mapuche), siempre designa la intensidad del color. Por ejemplo, en la taxonomía cromática mapuche, pelochods y pelokelü aluden a los colores naranja y rojo, donde el prefijo pelo significa "mayor luminosidad" (Alvarado, 2004).

Como indica el autor, los Mapuche relacionan muchas expresiones con la dualidad de la cosmovisión, por ejemplo la figura femenina y masculina, el día y la noche, la luz y su ausencia (Alvarado, 2004). En este caso se presenta el color blanco como luminosidad, la presencia de luz en un espacio determinado, versus el color negro como ausencia.

En cuanto al negro, Wekufii y sus huestes son seres de las tinieblas, de la no-luz. Por ende, son negros, no reflejan luz, la absorben; diríamos que más bien por extensión terminológica que por una cualidad de color. En la vestimenta, el negro es el color "original" es el color fundamental, sobre el que los demás colores se posan. Las prendas básicas son negras (kepan y chiripa) y se aceptan colores en sus márgenes. El fondo negro es la estabilidad, el color mas sólido. Así, la ambivalencia del significado del negro fluctúa entre su simbolización de lo destructivo la oscuridad y de lo estable el color negro, que está sumergido en un contexto de luz, "los hombres bajo el sol". Además, el negro en su significación de lo destructivo es a la vez opacidad; en cambio, al asumir una significación de lo estable es brillante. La esfera de wekufii es opaca; en la ropa de verdadera calidad la lana negra brilla. Esta ambigüedad del significado tiene al parecer un enlace de sentido, ya que tanto una significación como en la otra están por un sentido de lo que es fuerte y poderoso (de Arte, B. D. M. C. 2008).

Por su parte lig, el blanco en su materialidad es luz; la categorización de este color debe ser asociada necesariamente a la claridad, no en cuanto valor, sino porque es luz concreta, sólida. Este simboliza a la vida, a la existencia en su grado más sublime, en oposición a la oscuridad de la muerte. La luz blanca en determinados contextos no es de ninguna manera vida; figuras míticas nocturnas y letales son luz concentrada, fosforescentes. Es el caso de witranalwe y anchimallen, espíritus de la noche cargados de una luz enceguecedora, vestidos de blanco. La luz blanca de estos seres pertenece al dominio de la oscuridad, medio dominado por wekufii. Así, su luminosidad se carga de una significación diferente opuesta al sumergirse en un medio de tinieblas y muerte. (de Arte, B. D. M. C., 2008). 
El rojo o kelü es básicamente sangre de diferentes tipos, cuando adquiere valor significativo, siempre es sangre que fluye. La sangre que fluye por menstruación es una sustancia poderosísima, y lo es aún más la de la menarquia, con la cual "la" machi pinta su kultrún (Verniory, 1975). Dentro de la esfera de lo femenino es la materia germinadora de la vida, es la sustancia de la gestación e impregna toda matriz de vida humana. En la esfera de lo masculino, es impureza, lo que envenena por contaminación. El hombre evita este tipo de sangre y evidentemente no utiliza en su ropa los símbolos que se recubren con este ícono de color. El mundo masculino está impregnado de otro tipo de sangre que fluye, y es la sangre que emana de toda herida producto de la agresión (de Arte, B. D. M. C. 2008).

Existe una luz-calor necesariamente benigna, la amarilla o chods. El sol y la luz se representan icónicamente por el amarillo. Más que luz, es calor germinador de vida. Esta emana por encima de la bóveda celeste, es el calor del supramundo, dándose un nexo fortísimo con el azul ( $k a l f u)$, el color de la bóveda celeste Zijken asociado a su vez con el blanco de la luna y las estrellas. Todos forman una unidad semántica, de modo que son colores "transformacionales", su identidad es equivalente aunque no idéntica (Grebe, 1972) y se demuestra este principio en las banderas de muchi y en el ngillatun. (Arte, B. D. M. C., 2008). El amarillo se asocia persistentemente al oro, el metal de los dioses. En el wenumapu se habita en el oro, que es el mundo de la luz más brillante; tanto es así que milla (oro) y chods son palabras homónimas del referente oro y luz brillante.

En cuanto al verde o karü, se asocia directamente a la tierra, a una tierra muy especial; y para un pueblo fuertemente ligado a actividades agrícolas y ganaderas, una "tierra verde" es evidentemente una proyección ideológica de la abundancia y la prosperidad buscada. Donde lo verde, el mundo vegetal, que a la vez todo lo nutre, se realiza con gran profusión y de manera inalterable.

El azul o kalfu es un color positivo; simboliza, por lo general, el espacio celeste o el agua, dependiendo en que contexto se sitúe. Es considerado, por esta doble representación, de espacio sacro y líquido vital, de una gran importancia. La identidad cielo-agua es muy pronunciada y la frontera entre estos dos elementos es bastante difusa. En primer término, la bóveda celeste posee una ambigüedad de colores a partir de una dicotomía de elementos que, alternándose temporalmente, la pueden ocupar. Puede llenarse de luz, situación atmosférica que se denomina Ziflen (lifken: estar despejado, limpio) (De Augusta 1966); siendo el color de este estado el azul. Grebe (1972) se confunde, víctima de una analogía de su informante entre una situación climatológica, lifken, y su respectiva categoría de color, kalfu. Pero, al contener agua, se cubre de nubes, chiwai, siendo el color predominante el negro. (Arte, B. D. M. C., 2008).

\section{La Cosmovisión Mapuche representada en la artesanía}

Dentro del contexto en donde se desenvuelve la cultura Mapuche, es necesario definir la cosmovisión, parte fundamental de su origen como sociedad y pueblo originario. Para esto se presentan los siguientes relatos de autores que han desarrollado extensas investi- 
gaciones al respecto. Desde la visión de la cosmovisión Mapuche se puede identificar la identidad del pueblo originario, reflejada en sus prácticas sociales y religiosas, a los fines de este artículo el foco de interés son el color, la forma y su comunicación como tal. En efecto, según Mires (1992): "La cosmovisión es el todo. Es la armonía. Es el equilibrio. Es el hombre, naturaleza y lo que ocurre" (p. 120).

En las culturas originarias, tales como la mapuche, la dualidad S-O, desde el punto de vista epistemológico y ontológico, está constituida por elementos coherentes, representados en la correlación de pares de realidades multidimensionales. Por ejemplo, unidades tales como hombre-mujer, hombre-naturaleza, hombre-mujer-comunidad, hombre-mujer-comunidad-fuerzas naturales, seres sobre naturales y espirituales, van configurando realidades con caracteres hologramáticos (Izquierdo, 1999), que se van constituyendo en sistemas complejos. Debe entenderse que este carácter unitario de las culturas originarias, no se refiere a los dualismos epistemológicos ni ontológicos propios de la cultura occidental. En las culturas originarias es más propio hablar de un monismo epistemológico y ontológico, en cuya racionalidad el conocimiento se construye como un sistema de saberes subjetivo, intersubjetivo e interrelacionado (Quintriqueo, y Torres, 2013).

La religiosidad del pueblo Mapuche ha sido estructurada a través de complejas formas de comprensión del mundo, al configurar variados simbolismos, los que conforman un conjunto de creencias y rituales sagrados, que están presentes en la mayoría de los momentos de la vida cotidiana y del lenguaje de este pueblo. "Posiblemente en Chile sean pocos los grupos o sectores que manifiestan con tanta claridad como los mapuche, que su identidad, su ser se liga hasta confundirse con lo sagrado" (Foerster, 1995:93).

Los Mapuches conciben el cosmos como una serie de plataformas que aparecen superpuestas en el espacio. Dichas plataformas son todas de forma cuadrada y de igual tamaño. Fueron creadas en orden descendente en el tiempo de los orígenes, tomando como modelo la plataforma más alta, recinto de los dioses creadores. La agrupación de estas plataformas cuadradas define la ubicación de las tres zonas cósmicas: cielo, tierra e infierno (Grebe, Pacheco y Segura, 1972).

Las cuatro plataformas del bien, wenu mapu o meli ñom wenu, son el aposento ordenado y simétrico de los dioses, espíritus benéficos y antepasados. Ellas se oponen a las dos plataformas del mal, anka wenu y minche mapu, zonas oscuras, extrañas y caóticas en las cuales residen, respectivamente, los espíritus maléficos (wekufe) y los hombres enanos o pigmeos (laftrache) (Grebe, Pacheco y Segura, 1972).

La visión cósmica Mapuche es dualista y dialéctica: el wenu mapu contiene sólo al bien (tesis); el anka wenu y minche mapu representan sólo el mal (antítesis); y en la tierra coexisten el bien y el mal en una síntesis que no implica fusión, sino yuxtaposición dinámica. La verdadera polaridad tiende a la unión; y la conjunción de dos fuerzas opuestas es una condición necesaria para lograr el equilibrio cósmico dualista (Grebe, Pacheco, y Segura, 1972).

El universo Mapuche está orientado según los cuatro puntos cardinales, reconociéndose, por lo tanto, cuatro direcciones organizadas a partir del Este, lugar de la Cordillera de los Andes y región matriz de la presente concepción espacial. Consecuentemente, el ciclo solar diario parece ser decisivo en la elección de este punto de referencia, puesto que en el área andina el sol nace en la cordillera (Este) y se pone en el mar (Oeste). (Grebe, Pacheco y Se- 
gura, 1972). En efecto, según los cuatro puntos cardinales, se reconocen cuatro direcciones, de las cuales el este es el origen, desde donde nace el sol, trayendo consigo el comienzo de la vida. Todas estas expresiones se vislumbran en el trazado del kultrún, instrumento musical utilizado por la machi. "El kultrún representa de manera pragmática cómo el Mapuche se imaginó el cosmos, la Tierra, y el sistema solar en su conjunto” (Ñanculef 2006:5). Otra concepción asume también una interpretación témporo- espacial, vinculada a los movimientos que se realizan en la vida diaria. "Durante la celebración, la comida y la bebida se le pasa a los invitados siguiendo esta lógica circular de los movimientos” (Grebe1974:57).

Finalmente, se cuenta con la explicación colorista de la vida, llevada a un plano personal, plasmada en la vestimenta. Esta concepción llevada a las plataformas, se define en las cuatro superiores; el bien, el blanco y azul; la inmediatamente inferior, de transición, el rojo; la terrestre, donde están presentes todos estos matices; y la inferior, el mal, reflejado por el infierno, el negro. Así, los colores y sus gamas solo están presentes en la plataforma terrestre. (Rodríguez, y Saavedra, 2011).

La cosmovisión se define como un todo en la cultura Mapuche, y da pie al nacimiento de las expresiones artísticas que desarrollan, el diseño es representado en cada producto, ya sea de forma gráfica explícita, o simplemente con la simbología.

\section{Conclusiones}

A modo de conclusión, se puede visualizar en este artículo, que los emprendedores de etnia Mapuche han venido realizando sus creaciones, por medio de la artesanía. En éstos términos el desarrollo de la artesanía hizo conexión con lo que necesitaban en distintos periodos de tiempo, contextos sociales, económicos, incluso políticos. Lo anterior fue realizado con el fin de prestar un servicio utilitario a un grupo objetivo determinado, ya fuese en sectores familiares, o con el fin de realizar el trueque con los colonizadores.

También se puede apreciar en la construcción de datos en la investigación, que los emprendedores siguen desarrollando la artesanía en sus creaciones, cada uno de ellos crea una pieza original en un material determinado, y lo ofrece para comercializar.

Como se menciona, el trabajo artesanal se basa en la cosmovisión de la etnia Mapuche, y se plasma en distintos soportes; lana, cuero, plata, greda y madera. Materiales presentes en la selección del estudio.

La artesanía fue la forma inicial de comercialización de la etnia Mapuche, que permitió el intercambio de bienes materiales entre sectores sociales, y sobre todo hacia segmentos externos, por ejemplo con los colonizadores. Técnicas ancestrales que en la actualidad se siguen aplicando, y que nuevas generaciones mantienen la cultura artesanal como un patrimonio vivo.

\section{Lista de Referencias bibliográficas}

Aedo, A. W. (1992). Textilería mapuche, arte de mujeres (Vol. 3). Ediciones CEDEM.

Albuerne, I. Diaz, V. y Zárate (2008). Diseños indígenas Argentinos. Buenos Aires: Pearson Education. 
Alvarado, M. (2004). El lenguaje de los dioses: arte, chamanismo y cosmovisión indígena en Sudamérica. Biblos: Buenos Aires.

Chávez, J. F. (2013). "La ocupación de la Araucanía y la pérdida de la platería en manos mapuches. Finales del siglo XIX y primeras décadas del XX”. Revista de Indias, 73(259), 825-854.

de Arte, B. D. M. C. (2008). Boletín del Museo Chileno de Arte Precolombino, vol. 13, núm. 1, 2008 Museo Chileno de Arte Precolombino Santiago, Chile. Boletín del Museo Chileno de Arte Precolombino, 13(1).

Fiadone, A. (2007). Simbología Mapuche. El territorio Tehuelche.Buenos Aires: Maizal Ediciones.

García, F. J. F., y Cañizares, S. M. S. (2010). "Análisis del perfil emprendedor: una perspectiva de género". Estudios de economía aplicada, 28(3), 696.

Gedda, M. (2010) Patrimonio de la Araucanía - Chile. Manual de interpretación y puesta en valor. Ediciones sede regional Villarrica Pontificia Universidad Católica Villarrica: Chile.

Grebe, M. E., Pacheco, S., y Segura, J. (1972). “Cosmovisión mapuche”. Cuadernos de la realidad nacional, 14, 46-73.

Guebalmar, G. R. (2014). "El motivo del orante arrodillado y el mito de Trentren y Kaikai”. Lenguas y Literaturas Indoamericanas, (4).

Hernández. I. (2003) Autonomía o ciudadanía incompleta. El pueblo Mapuche en Chile y Argentina. Pehuén editores: Chile.

Kradolfer, S. (2000). "Muy lindos látigos tenés vos": la artesanía del cuero entre los mapuche argentinos”. Bulletin-Société suisse des américanistes, (64-65), 61-68.

Mastandrea, M. (2008) Arte Mapuche. Editorial Guadal: Buenos Aires, Argentina.

Navarro Hoyos, S. (2017). La artesanía como industria cultural: Desafíos y oportunidades. In Obtenido de Pontificia Universidad Javeriana. Departamento de Diseño, Facultad de Arquitectura y Diseño, Bogotá DC, Colombia, SA: Recuperado de https://www. fessociologia. com/files/congress/12/papers/3519.pdf .

Pérez, T. Catriquir, D. Hernández Sallés, A. (2007) Patrimonio cultural Mapunche. Derechos Lingüísticos y patrimonio cultural Mapunche. Volumen I. Editorial UC Temuco. Temuco, Chile.

Peyser, A. (2003) Desarrollo, Cultura e Identidad. El caso del mapuche urbano en Chile. Elementos y estrategias identitarias en el discurso indígena urbano. Diffusion universitaire CIACO: Belgique.

Quintriqueo, S., y Torres, H. (2013). “Construcción de Conocimiento Mapuche y su relación con el Conocimiento Escolar”. Estudios pedagógicos (Valdivia), 39(1), 199-216.

Rodríguez, C., y Saavedra, A. (2011). "Cosmovisión mapuche y manifestaciones funerarias. Si Somos Americanos" Revista de Estudios Transfronterizos, 11(2).

Roselló, J. G. (2009). “Tradición tecnológica y variaciones técnicas en la producción cerámica mapuche/Technological tradition and technical changes in the mapuche pottery production". Complutum, 20(1), 153.

Salas, E. (1997) Araucanía Mitológica. Tradiciones, ritos y pinturas. Imprenta Austral, Temuco Chile.

Serrano, M. T. E. (2004). Creatividad: definiciones, antecedentes y aportaciones. Revista digital universitaria, 5(1), 1-17. 
Sondereguer, C. (2003). El diseño mítico en Amerilandia. Buenos Aires: Perar Ed. Vinagre, A. (2017). ¿ Terrorismo en La Araucanía?

Vera, R. Aylwin, J. Coñuecar, A. Chihuilaf, E. (1994) El despertar del pueblo mapuche. Nuevos conflictos, viejas demandas. LOM Ediciones: Santiago de Chile.

Wong. W. (1995). Fundamentos del diseño. Gustavo Gili. S.A.: Barcelona.

Wong. W. (1988). Principios del diseño en color. Gustavo Gili. S.A.: Barcelona.

Yáñez, N. Soto, M. Vera, F. Fajreldin, V. Vega, C. (1994) Pueblos indígenas olvidados y extintos. LOM Ediciones: Santiago de Chile.

Zúñiga, C. (2011) Fragmentos de historia regional. La araucanía del siglo XX. Ediciones Universidad de la Frontera: Temuco, Chile.

\begin{abstract}
The following article presents an extract of the research carried out to qualify for the degree of Doctor of Design at the University of Palermo, in Buenos Aires. Argentina. Referred to the development of Mapuche entrepreneurs craftsmanship in La Araucanía region, Chile. This article studies the relationship between designs with graphic motives in the elaboration of handmade products. Materials such as leather, silver, wool, ceramic clay and wood are some of the study's objects that are presented in proposals of entrepreneurs who design graphic motifs.
\end{abstract}

Keywords: Entrepreneurship - Design - Mapuche - Ethnicity - Crafts

Resumo: $\mathrm{O}$ artigo a seguir apresenta um extrato da pesquisa realizada para se qualificar para o grau de Doutor em Design na Universidade de Palermo, em Buenos Aires. Argentina Referiu-se ao desenvolvimento de artesanato em empresários mapuche na região de La Araucanía. Chile Uma abordagem sobre a relação direta que tem o trabalho em design com motivos gráficos nos formatos que permitem a elaboração de produtos artesanais. Materiais como couro, prata, lã, argila cerâmica e madeira são alguns dos objetos de estudo que são apresentados em propostas de empreendedores que desenham motivos gráficos neles.

Palavras chave: Empreendedorismo - Design - Mapuche - Etnicidade - Artesanato

[Las traducciones de los abstracts fueron supervisadas por el autor de cada artículo] 